\title{
Raciocínio condicional: a conclusão depende do conhecimento armazenado na memória
}

\author{
Goiara Mendonça de Castilho \\ Gerson Américo Janczura \\ Universidade de Brasília
}

\begin{abstract}
Resumo
Exemplos alternativos armazenados na memória são fundamentais para a correta interpretação da proposição condicional "Se P, então Q". Esse artigo propõe que a força associativa e o tamanho da categoria semântica têm um papel crítico na ativação de modelos alternativos envolvidos neste tipo de raciocínio. Adicionalmente, quanto mais modelos alternativos, justificados em função do tipo de inferência, maior a dificuldade da tarefa, dada a sobrecarga de informação na memória de trabalho. Dois Experimentos testaram essas hipóteses, sendo que os resultados do Experimento 1 confirmaram efeitos significativos da força associativa, tamanho da categoria, tipo de inferência e interação entre fatores. O Experimento 2 evidenciou que os efeitos de força associativa são contextos-dependentes devido à redução do campo semântico. Esses achados são consistentes com a hipótese de que o conhecimento armazenado na memória tem um papel importante no raciocínio condicional.
\end{abstract}

Palavras-chaves: raciocínio condicional; modelos mentais; força associativa; tamanho da categoria.

\begin{abstract}
Conditional reasoning: the conclusion depends on the knowledge stored in memory.

Alternative examples stored in memory are fundamental for the correct interpretation of the conditional proposition "If $\mathrm{P}$, then Q". This paper proposes that associative strength and set size of semantic categories have a critical role in activation of alternative models. Also, the more the number of alternative models generated as a function of inference type, the greater the difficulty of the task due to information overload in working memory. Experiment 1 confirmed significant effects of associative strength, category set size, type of inference and interaction effects. Experiment 2 showed that the effects of associative strength are context-dependent determined by the reduction of semantic field. These findings are consistent with the hypothesis that knowledge stored in memory plays an important role in conditional reasoning.
\end{abstract}

Keywords: conditional reasoning; mental models; associative strength; category set size

$\mathrm{E}$ mbora a lógica formal, ou seu equivalente no campo da psicologia do raciocínio, a lógica mental (Bonatti, 1994; Braine, 1978; Rips, 1983), proponha o uso de regras formais para o raciocínio humano, dados empíricos revelam que nem sempre as pessoas seguem tais regras (Barrouillet, Gaufroy, \& Lecas, 2008; Johnson-Laird \& Byrne, 2002; Oaksford \& Chater, 1994; Wason, 1968). Por exemplo, quanto ao aspecto formal, a implicação ou regra condicional "Se P, então Q" é caracterizada por quatro tipos de inferências, sendo duas válidas e duas inválidas. Para ilustrar esses conceitos, tomemos um exemplo concreto da regra condicional "Se P, então Q": "Se é inverno [proposição P], então faz frio [proposição Q]". Qual conclusão se segue à afirmativa "É inverno"? Nesse caso, é válida a conclusão "faz frio", pois a condição para que isso ocorra foi satisfeita. Essa inferência é denominada modus ponens (MP) e é pautada na relação de que, uma vez posto o antecedente (a afirmação da proposição P), posto está o consequente, ou seja, afirmada está a proposição Q. O segundo tipo de inferência válida, a inferência modus tollens (MT), decorre da negação do consequente. Assim, tolhida a consequência, ou seja, negandose a proposição "Q" - "Não faz frio" - tolhida está a condição ou proposição antecedente. Destarte, conclui-se logicamente que "Não é inverno". Os outros dois casos incluem as falácias lógicas baseadas na afirmação do consequente (AC) e na negação do antecedente (NA). No primeiro caso, ao afirmar que "Faz frio", não se pode concluir que seja inverno dado que a regra não exclui outras possibilidades. Por exemplo, pode ser que seja outono e ainda assim fazer frio. Por fim, a inferência NA tem, igualmente, caráter indeterminado. Nesse caso, ao negar-se a proposição antecedente, "Não é inverno", não se pode concluir se faz frio ou não.

Contudo, a despeito da lógica subjacente a cada argumento, um padrão sistemático tem sido observado em tarefas com condicionais: a produção quase ubíqua das inferências válidas 
MP, seguidas das inferências AC (inválidas) e a quase absoluta rejeição (ou não produção) das inferências válidas MT (Oaksford \& Chater, 1994). Todavia, manipulações experimentais podem conduzir a variações nesse padrão (De Neys, Schaeken, \& d'Y dewalle, 2002, 2005a, 2005b). Por exemplo, se a regra acima é apresentada a uma pessoa que vive no nordeste do Brasil, é possível que ela rejeite a inferência MP. Essa rejeição poderia ser justificada pela lembrança de que, mesmo no inverno, faz calor no nordeste brasileiro. Por outro lado, se a mesma regra fosse apresentada a uma pessoa que vive no sul do Brasil, a inferência AC poderia ser adequadamente rejeitada, dada a reconhecida probabilidade de fazer frio em outras estações do ano. Nesses casos, os processos de raciocínio seriam antes justificados pela experiência do indivíduo do que pelo uso de regras formais. $\mathrm{O}$ presente artigo focaliza essas questões e sustenta que a compreensão dos processos de raciocínio necessita incluir as relações entre os conceitos associados na memória semântica, evidenciando uma interação entre o conhecimento coloquial e o raciocínio condicional.

O reconhecimento dessa interação perpassa por muitas posições teóricas no campo. Por exemplo, Johnson-Laird e Byrne (2002) ressaltam que o raciocínio condicional é influenciado pelo conhecimento que temos porque pensamos muito frequentemente a partir das relações "se, então". Ilustrando, podemos pensar que "se há uma crise econômica mundial, então a inadimplência aumentará" ou "se há uma pandemia de gripe, então os aeroportos adotarão procedimentos mais rigorosos". As situações em que os indivíduos adotam uma relação "se, então" são muito cotidianas e refletem, de acordo com os autores, a organização do conhecimento de mundo que as pessoas têm. Ou seja, o raciocínio condicional seria influenciado por processos semânticos, um argumento que tem como proposição central o uso de modelos mentais como representações das relações capturadas entre " $P$ " e "Q" (Johnson-Laird \& Byrne, 2002; Jonhson-Laird, Byrne, \& Schaeken, 1992). Por exemplo, retomando a fórmula condicional "Se é inverno, então faz frio", tem-se o seguinte modelo mental:

Inverno

Frio

O modelo acima permitiria a inferência MP, derivada diretamente da relação modelada acima. Contudo, de acordo com Johnson-Laird et al. (1992), uma conclusão pode ser refutada se houver contraexemplos adicionados ao conjunto de modelos, um processo denominado fleshing-out. Assim, no caso acima, a inserção de um contraexemplo provável poderia concorrer para a rejeição da inferência válida MP. Ilustrando:

$\begin{array}{ll}\text { Inverno } & \text { Frio } \\ \text { Inverno } & \text { Calor }\end{array}$

É interessante observar que, nesse caso, a inferência MP seria provavelmente rejeitada, dado que o segundo modelo falseia a contingência estabelecida pela relação lógica entre as proposições "P" e "Q". Vale lembrar que no caso dos condicionais, é exigido que as inferências válidas MP e MT sejam produzidas e que as falácias lógicas AC e NA sejam rejeitadas. Segundo Goodwin e Johnson-Laird (2005), os indivíduos são capazes de apreender tais relações lógicas inerentes ao condicional em função dos tipos de modelos gerados e do conhecimento subjacente às relações mapeadas nesses modelos. Por exemplo, para que um indivíduo rejeite a inferência AC seria necessário considerar alternativas para a ocorrência de "Q". Assim, o conjunto de modelos gerados incluiria as situações nas quais "Q" ocorre tanto na presença quanto na ausência de "P" ("p q" e " $\neg p$ q"), respectivamente). Ilustrando, isso ocorreria se fosse adicionada ao conjunto de modelos acima uma relação que evidenciasse a ocorrência de uma estação diferente do inverno na qual se fizesse frio. Adicionalmente, Johnson-Laird et al. (1992) sustentam que a inserção da relação " $\neg p \neg q$ " (isto é, "não é inverno, não faz frio") é fundamental para a correta interpretação dos condicionais, totalizando três modelos: "p q", “ $\neg \mathrm{p}$ " " e " $\neg p \neg q$ ". Caso diferente ocorreria se a regra expressasse a seguinte relação bicondicional "Se e somente se for inverno, faz frio", onde não haveria a possibilidade de outra condição aceitável, além do inverno, para que o frio ocorresse. Segundo Johnson-Laird et al. (1992), os modelos subjacentes a uma interpretação bicondicional seriam dois, e incluiriam as relações "p q" e " $\neg \mathrm{p} \neg \mathrm{q}$ ".

Consequentemente, muitos erros no raciocínio condicional poderiam ser justificados pela interpretação incorreta da regra “Se P, então Q", que seria caracterizada pela não percepção do caráter indeterminado das inferências AC, principalmente, e NA. No que tange à proposta de Johnson-Laird et al. (1992), tal padrão de interpretação apresentaria estreita correspondência com os modelos gerados a partir das relações mapeadas entre "P" e "Q".

A hipótese sobre os efeitos da interpretação do raciocínio condicional foi apontada anteriormente (Bucci, 1978; Pollard, 1982; Thompson, 1995), sendo considerado fundamental o conhecimento armazenado de contraexemplos alternativos. Thompson (1995) salienta que se houver antecedentes alternativos, as pessoas tenderão a rejeitar, corretamente, as inferências AC e NA, dada a relação de não-necessidade percebida. A regra "Se é uma cor de cabelo, então é o azul" ilustra essa relação, pois, as pessoas poderiam perceber mais facilmente que muitas coisas, que não a cor de cabelo, poderiam ser azuis. De acordo com Thompson, tal percepção incorreria em uma interpretação correta do condicional. Já a ocorrência de consequentes alternativos contribuiria para uma relação de nãosuficiência percebida e, em consequência, Thompson sustenta o declínio da produção ou aceitação das inferências válidas MP e MT. Nesse caso, Thompson usa o termo "interpretação condicional reversa" para indicar a produção das inferências AC e NA e a rejeição das inferências MP e MT. Nessa linha de argumentação, vários achados empíricos têm apresentado um conjunto de evidências robustas quanto aos efeitos do conhecimento sobre o raciocínio a partir das relações mapeadas entre "P" e "Q" (Barrouillet \& Lecas, 1998; Byrne, 1989; Cummins, 1995; Cummins, Lubart, Alksnis, \& Rist, 1991; De Neys et al., 2002, 2005a, 2005b; Evans, Clibbens, \& Rood, 1995; Evans \& Twyman-Musgrove, 1998; Quinn \& Markovits, 1998).

Entretanto, dois aspectos devem ser observados nestes estudos: primeiro, a discordância sobre os processos subjacentes à inserção de exemplos alternativos. Schroyens e Schaeken (2008), por exemplo, defendem a busca ativa e intencional de 
contraevidências que permitam falsear uma conclusão putativa inicial em oposição à inserção de contraexemplos ativados passivamente, segundo os autores, em função de associações semânticas pré-existentes. Consonante como esses argumentos, outros trabalhos sugerem que a busca ativa de contraexemplos é gerenciada pelo executivo central, componente da memória de trabalho envolvido no processamento, ativação, manutenção e monitoramento dos processos de busca (De Neys et al., 2005a, 2005b; García-Madruga, Gutiérrez, Carriedo, Luzón, \& Vila, 2007). Em oposição, Cummins (1995) e Cummins et al. (1991) sugerem que os processos de busca envolvem ativação automática de contraexemplos. Estudos mais recentes têm evidenciado que tais processos automáticos são pouco influenciados pela sobrecarga da memória trabalho. Por exemplo, em um estudo de De Neys et al. (2005a), os participantes deveriam gerar contraexemplos para condicionais ao mesmo tempo em que executavam tarefas motoras que variavam em complexidade. Os resultados mostraram que a geração de contraexemplos mais acessíveis à memória, portanto, ativados sem esforço, era pouco afetada pela natureza da tarefa motora. Esta questão é fundamental para a compreensão dos mecanismos de raciocínio e evidenciaria a participação dos processos subjacentes à inserção de contraexemplos ao conjunto de modelos mentais, como observa Johnson-Laird (2001).

O segundo aspecto relevante a ser observado relaciona-se ao escopo de fenômenos investigados nos estudos anteriores. Apesar de tais estudos terem investigado de forma independente a contribuição dos efeitos do tamanho do grupo de associadas (Cummins et al., 1991) ou da força associativa (Quinn \& Markovits, 1998), nenhuma tentativa sistemática articulou estas variáveis à hipótese de modelos mentais, modos interpretativos e processos de busca da informação armazenada na memória semântica. Essa lacuna é evidenciada, principalmente, no campo da investigação experimental do raciocínio por pesquisadores brasileiros. Assim, o presente estudo objetiva contribuir para a compreensão dos mecanismos subjacentes à construção dos modelos mentais quanto à consideração de contraexemplos (fleshing-out), o aspecto chave que modelaria a interpretação dos condicionais. Esta abordagem permitiria a compreensão do raciocínio condicional considerando a estrutura do conhecimento armazenado na memória. No que concerne ao problema dos processos de inserção de contraexemplos sugere-se, diferentemente de Schroyens e Schaeken (2008), a participação da ativação de redes semânticas, geralmente inevitáveis e inacessíveis ao controle do indivíduo. Similarmente aos argumentos de Cummins (1995), propõe-se que, dentre os fatores que poderiam deflagrar a ativação de exemplos alternativos na rede semântica, destacam-se força associativa e o tamanho da categoria semântica. A força associativa se relaciona à probabilidade de ocorrência de um evento (Janczura, 1996, 2005; Nelson, Schreiber, \& McEvoy, 1992). Ou seja, uma palavra é mais fortemente associada à outra quando ela é mais provavelmente evocada na presença da primeira palavra. Ilustrando, no caso da categoria "cor de cabelo", seria mais provável a produção de "louro" do que "azul", porque o primeiro estaria mais fortemente associado à categoria. Esta evocação é automática devido à ocorrência de processos de ativação implícitos na memória (Nelson, Mckinney, Gee, \& Janczura 1998). Ou seja, mesmo que os indivíduos se engajem na busca de evidências que poderiam falsear suas conclusões, conforme sugerem Schroyens e Schaeken (2008), na acepção da ativação automática, as proposições "P" e "Q" presentes na fórmula condicional, disparariam o acesso automático às informações na memória, caracterizando efeitos de pré-ativação (priming) que escapariam ao monitoramento consciente dos participantes. Adicionalmente, vários estudos de Nelson (e.g., Nelson, Fisher, \& Akirmak, 2007; Nelson, Dyrdal, \& Goodmon, 2005; Nelson, McEvoy, \& Pointer, 2003; Nelson et al.,1998) demonstraram que palavras associadas pelas experiências cotidianas compõem conjuntos semânticos de diferentes tamanhos e diferentes padrões de interconexões, caracterizando uma estrutura associativa, cuja arquitetura teria papel significativo na recuperação da informação.

Nós argumentamos que a estrutura associativa pode ajudar a compreender a interação entre memória e raciocínio. Pretendese oferecer, mais especificamente, uma resposta alternativa à questão de Johnson-Laird (2001) e Schroyens e Schaeken (2008) quanto aos fatores que facilitariam os processos de busca e ativação de contraexemplos. Nossa hipótese de trabalho sustenta que a acessibilidade da informação na memória, indexada através da força associativa e do tamanho da categoria, influencia a construção de modelos mentais alternativos em tarefas de raciocínio (processos de fleshing-out), e por extensão, os modos interpretativos (padrão condicional e bicondicional). Teoricamente, itens mais fortemente associados contribuiriam para maiores aceitações dos quatro tipos de inferências. Isto ocorreria devido à maior probabilidade de ativação de itens fortemente associados quando comparados aos itens mais fracamente associados. Considerando essa relação, os modelos mentais gerados sugeririam uma interpretação bicondicional (i.e., Se e somente se P, então Q), onde uma forte força associativa levaria os participantes a avaliar a relação entre "P" e "Q" como necessária e suficiente. Por outro lado, itens mais fracamente associados facilitariam a rejeição dos quatro casos lógicos em função da ativação automática de itens mais fortemente associados à pista, no caso, a proposição antecedente. Por exemplo, se um participante recebesse a regra "se é uma cor de cabelo, então é o azul", a pista "cor de cabelo" poderia ativar o "louro", uma cor mais fortemente associada à categoria "cor de cabelo" levando não apenas a rejeição da inferência MP como também dos outros três casos lógicos.

Quanto ao efeito do tamanho do grupo de associadas, Nelson et al. (1998) mostraram que a taxa de lembrança na recuperação com pista intralista é inversamente proporcional ao tamanho do conjunto de palavras associadas, dado que quanto mais itens existem num conjunto, maior é a probabilidade de competição entre estes itens e o alvo a ser lembrado. Nelson et al. (1998) sugerem a existência de competições entre itens porque os processos de busca ou ativação seriam automáticos, criando representações implícitas. Entretanto, enquanto na pesquisa experimental sobre memória a competição entre palavras pode comprometer aquilo que é lembrado, no campo do raciocínio a competição pode ser a chave para se compreender porque as pessoas produzem conclusões válidas ou falaciosas. Ou 
seja, a competição decorrente da ativação automática de itens associados poderia explicar a influência do tamanho do grupo de associadas sobre o raciocínio onde quanto maior for o conjunto semântico, maior será a chance de exemplos alternativos serem considerados. Em termos práticos, o aumento de exemplos alternativos referentes à categoria semântica poderia facilitar a apreensão da indeterminação inerente às inferências $\mathrm{AC}$ e NA, indicando uma interpretação condicional. Por outro lado. e em consonância com o estudo de Cummins et al. (1991), a ativação de exemplos alternativos tenderia a obstaculizar a aceitação das inferências MP e MT. Além disto, espera-se que os itens mais fortemente associados inibam a ativação de contraexemplos quando se referirem, principalmente, a conjuntos com um menor número de itens. Isto ocorreria porque a ativação do item fortemente associado estabeleceria limites à amplitude de ativação da rede semântica. Em termos práticos, isso significa que exemplos mais fortemente associados a categorias pequenas otimizariam as inferências válidas MP e MT, mas também concorreriam para uma maior incidência das inferências inválidas AC e NA. Por outro lado, quanto mais fraca for a força associativa e quanto maior for o conjunto de itens pertencentes à categoria, mais chance teria o participante em perceber a indeterminação das inferências inválidas. Isto aconteceria porque itens fracos e fortemente associados seriam ativados, sendo os últimos considerados contraexemplos mais plausíveis na percepção do participante.

Ademais, o presente estudo permitiu apreciar uma das principais predições referentes à construção e manipulação de modelos mentais que se referem à relação entre a sobrecarga da memória de trabalho e a dificuldade em se produzir conclusões no contexto do raciocínio condicional (Johnson-Laird, 2001; Klauer \& Oberauer, 1995; Legrenzi, Girotto, \& Johnson-Laird, 1993). Assim, quanto mais modelos são gerados na memória de trabalho para se evitar conclusões falaciosas, mais difícil se tornam estas tarefas, potencializando a ocorrência de erros, principalmente em relação às inferências que exigem um maior número de modelos, tais como as inferências $\mathrm{AC}$ e MT. Essa expectativa alia-se ao reconhecimento que é oneroso para a memória de trabalho, especificamente para o executivo central, processar a informação e manipular os modelos que possam ser gerados pela ativação automática da informação de longo-prazo (García-Madruga et al., 2007).

As expectativas delineadas acima foram testadas em dois experimentos. No Experimento 1 foram avaliados os efeitos da força associativa e do tamanho das categorias sobre o desempenho em tarefas de raciocínio condicional. Adicionalmente, o tipo de inferência foi tratado como variável independente, considerando-se as previsões de Johnson-Laird (2001) e Klauer e Oberauer (1995) quanto à relação entre a quantidade de modelos e dificuldade da tarefa. O Experimento 2 investigou os efeitos de força associativa e tipos de inferências sobre condicionais inseridos em contextos específicos que restringem o campo semântico.

\section{Experimento 1}

O objetivo desse experimento foi evidenciar que os processos subjacentes ao raciocínio condicional, no que tange à consideração de contraexemplos, podem ser compreendidos em função da ativação de itens semanticamente relacionados. De acordo com o modelo de Nelson et al. (1998, 2007), é esperado que o padrão de ativação seja fortemente influenciado pela força associativa e o tamanho do conjunto de associadas. Itens fortemente associados inibiriam a busca de contraexemplos e categorias com um maior número de itens facilitariam a busca por exemplos alternativos. Assim, quando os itens forem fortemente associados às categorias com um menor conjunto de associadas, os padrões inferenciais seriam caracterizados por uma interpretação bicondicional, respaldada na relação de necessidade e suficiência percebidas. Por outro lado, a percepção do caráter indeterminado das inferências AC e NA seria facilitado pela presença de itens mais fracamente associados e categorias com um maior conjunto de membros associados. A percepção da não-necessidade responderia por este padrão, dado que a inserção de contraexemplos seria facilitada pela existência de um maior número de possibilidades alternativas. Além disso, inferências que requerem múltiplos modelos (i.e., AC, NA e MT) serão mais difíceis, produzindo um menor número de acertos.

\section{Método}

\section{Participantes}

Participaram do estudo, voluntariamente, 66 alunos com média de idade de 27,8 anos $(D P=8,96)$ de curso noturno de graduação em Psicologia e sem qualquer treinamento em lógica formal. Os participantes eram de ambos os sexos, sendo mulheres em sua maioria $(78,8 \%)$.

\section{Delineamento}

Fatorial misto $2 \times 2 \times 4$, sendo a variável força associativa (forte e fraca) manipulada entre-sujeitos, e as variáveis tamanho do grupo de associadas (grande e pequeno) e tipos de inferências (MP, AC, NA e MT) manipuladas intra-sujeitos. A variável dependente correspondeu aos acertos na tarefa com problemas condicionais.

\section{Materiais}

Para elaborar o conjunto de 48 regras condicionais aplicadas a cada participante, foram utilizadas 12 categorias semânticas, sendo 6 grandes e 6 pequenas, cada qual com um membro forte e um fracamente associado, selecionados das normas de associação livre de Janczura (1996). Os critérios de seleção dos estímulos em função do tamanho da categoria e da força associativa seguiram as sugestões de Nelson et al. (1998), sendo que categorias grandes tiveram um número médio de 19 itens $(D P=1,47)$ e as pequenas, 7 itens em média, $(D P=2,23)$. Os exemplos fortemente associados às categorias grandes e pequenas apresentaram, respectivamente, uma força associativa média igual a $21 \%(D P=2,04)$ e $22 \%(D P=1,63)$. A força associativa média dos exemplos fracamente associados era $1 \%$ $(D P=0,41)$ para as categorias grandes e $2 \%$ para as categorias pequenas $(D P=1,6)$. Para cada uma das 12 categorias, foram elaborados 4 tipos de inferências (MP, AC, NA e MT) ou regras condicionais no formato "Se é um(a) P, então deve ser o(a) Q", 
onde 'P' referia-se a uma categoria semântica e a proposição 'Q' incluiu um membro associado à categoria, sendo que para metade dos participantes 'Q' incluiu um membro fortemente associado à categoria e, para a outra metade, um membro fracamente associado. Para cada problema, houve três alternativas dado um caso lógico. O exemplo abaixo ilustra uma inferência modus ponens:

Se é uma raça de cachorro, então deve ser o pastor alemão. É uma raça de cachorro. O que deve ser concluído?

a) deve ser o pastor alemão

b) não deve ser o pastor alemão

c) não é possível concluir algo

A palavra "deve" foi introduzida na regra e na pergunta com o objetivo de enfatizar tanto o caráter normativo da frase condicional quanto a necessidade de uma conclusão sobre a qual não deviam pairar dúvidas. Para cada nível da variável força associativa foram elaboradas 3 ordens randômicas.

\section{Procedimentos}

Cada participante recebeu um bloco de papel com as regras e opções impressas. A tarefa dos participantes foi julgar qual seria a conclusão apropriada na tarefa lógica de múltipla escolha. As instruções foram lidas pelo experimentador, sendo enfatizado que as conclusões deveriam se pautar na relação estabelecida entre as proposições "P" e "Q" e na informação adicional (afirmação ou negação dos antecedentes e consequentes). Os dados foram coletados em dois dias consecutivos em salas de aula com grupos de alunos. O tempo foi livre para a realização da tarefa.

\section{Resultados e Discussão}

Os resultados evidenciaram o efeito significativo da força associativa $(F(1,64)=11,639, M S=43,184, p=0,001)$, onde as médias de acertos para associações fortes e fracas foram, respectivamente, 2,51 e 1,94, sendo que itens mais fortemente associados levaram a um maior número de acertos quanto às inferências MP e MT, conforme era esperado. Adicionalmente, o $d$ de Cohen indicou um efeito de magnitude 0,30 para esse fator, ou seja, há cerca de $30 \%$ de chance de se reproduzir os efeitos para esse fator, sendo, portanto, pequena a variação entre a distribuição dos escores.

O tamanho do grupo de associadas também produziu um efeito significativo $(F(1,64)=14,959, M S=15,684, p$ $=0,000)$, sendo observado um maior número de acertos em relação às categorias pequenas (Média $=2,40$ ) em relação às categorias grandes (Média $=2,06$ ). Para esse fator, o $d$ de Cohen indicou um pequeno efeito de 0,33. O tipo de inferência influenciou significativamente os julgamentos dos participantes, $F(3,192)=50,653, M S=252,942, p=0,000$. As médias de acertos para as inferências MP, MT, AC e NA foram, respectivamente, $3,97,2,67,0,88$ e 1,39. O teste de Tukey ( $D$ $=0,68, p<0.05)$ evidenciou que todas as diferenças entre as médias foram significativas, exceto entre as médias de acertos para as inferências AC e NA. O teste $d$ de Cohen indicou um pequeno tamanho do efeito do tipo de inferência $(d=0,44)$. Esse resultado é consistente com a produção de dois modelos e com uma interpretação bicondicional, caracterizada pela percepção de relações necessárias e suficientes entre as proposições "P" e "Q" (Thompson, 1995). A interação entre força associativa, tamanho do grupo de associadas e tipo de inferência também foi significativa, $F(3,192)=6,858, M S=8,164, p=0,000$. As médias de acertos e desvios padrões para esta interação podem ser observadas na tabela 1 .

O teste de Tukey $(D=1,16, p<0.05)$ evidenciou que as diferenças entre as médias de acertos para as inferências MP foram significativas em função da força associativa, sendo observado um maior número de acertos para as categorias com membros fortemente associados. A menor taxa de acertos das inferências MP com itens fracamente associados é consistente com a ativação de exemplos alternativos, provavelmente membros mais fortemente associados às categorias. Se por um lado este resultado indica um forte viés categórico, por outro

Tabela 1

Médias de acertos e desvios padrões (DP) para a interação entre tipo de inferência (Modus Ponens [MP], Modus Tollens [MT], Afirmação do Consequente [AC], Negação do Antecedente [NA]), força associativa (Forte, Fraca) e tamanho da categoria (Grande, Pequena)

\begin{tabular}{llrlrcrcrc}
\hline \multirow{2}{*}{$\begin{array}{c}\text { Força } \\
\text { Associativa/Tipo de } \\
\text { Inferência }\end{array}$} & \multicolumn{2}{c}{ MP } & \multicolumn{2}{c}{ MT } & \multicolumn{2}{c}{ AC } & \multicolumn{2}{c}{ NA } \\
\cline { 2 - 9 } & Média & DP & Média & DP & Média & DP & Média & DP \\
\hline Forte/Grande & 4,30 & 1,85 & 2,21 & 2,10 & 1,06 & 1,73 & 1,24 & 1,73 \\
Forte/Pequena & 4,61 & 1,87 & 2,64 & 1,91 & 0,97 & 1,55 & 1,12 & 1,47 \\
Fraca/Grande & 3,51 & 1,82 & 1,70 & 1,83 & 0,55 & 1,09 & 1,88 & 2,04 \\
Fraca/Pequena & 3,45 & 1,52 & 2,18 & 1,73 & 0,94 & 1,22 & 1,33 & 1,59 \\
\hline
\end{tabular}

lado, a manipulação dos fatores força associativa e tamanho da categoria elevou a taxa de acertos das inferências MT a um nível superior aos valores geralmente encontrados na literatura (Evans, 1993; Oaksford \& Chater, 1994). Os dados apontam efeitos mais robustos da variável força associativa em relação às inferências MP e MT, sugerindo interpretações bicondicionais, pautadas na percepção de relações necessárias e suficientes (Thompson, 1995) que, por sua vez, podem ter sido realçadas pelas relações de pertencimento típicas de situações de categorização. As expectativas em relação às inferências AC e NA não foram confirmadas. Uma explicação para esse resultado se relaciona ao conhecimento prévio que as pessoas têm sobre categorias conceituais, onde pode parecer um contrassenso a indeterminação da inferência $\mathrm{AC}$, uma vez que 
o membro integrante da proposição "Q" é parte constituinte da categoria inclusa na proposição "P". Tal viés descreve um contexto no qual o padrão de ativação se espalha, mascarando quase completamente qualquer percepção da relação formal estabelecida pelos operadores "se, então", e evidenciando uma significativa influência de variáveis semânticas sobre o raciocínio. Isto sugere que a força associativa e o tamanho do conjunto são variáveis sensíveis a diferentes contextos. $\mathrm{O}$ experimento 2 investigou esta possibilidade.

\section{Experimento 2}

No experimento anterior, as associações semânticas foram mapeadas em um contexto demarcado por relações de pertencimento e de espectro mais amplo, sendo os efeitos da força associativa observados mais consistentemente. Entretanto, Janczura (2005) salienta que o contexto funcionaria como uma pista complexa, ativando na memória os elementos mais apropriados ou condizentes com a situação, delimitando, assim, o campo semântico. Por exemplo, as possibilidades de respostas para a pista "raça de cachorro" podem incluir vários membros, alguns mais prováveis de serem citados do que outros. Por outro lado, o conjunto de respostas associadas à pista contextual "raça de cachorro que vive em apartamento" reduz as possibilidades a serem consideradas e demarcam de forma mais definida o campo semântico referente à pista.

Dessa forma, os experimentos 1 e 2 se diferenciam quanto ao escopo de ativação dentro do campo semântico, sendo o Experimento 1 mais amplo e inespecífico e o Experimento 2 mais restritivo e específico. Isto considerado, quais seriam os padrões de ativação de contraexemplos em contextos mais restritivos? Ou seja, como o contexto circunscreve tais padrões, e por extensão, como afeta a compreensão dos condicionais? Considerando a redução do campo semântico, este experimento prevê que contextos que incluam elementos mais fortemente associados levarão a uma interpretação bicondicional (i.e., aceitação dos quatro tipos de inferências) por inibirem a ativação de exemplos alternativos mais fracamente associados. Em consequência, a interpretação do condicional seria respaldada pela percepção das relações entre "P" e "Q" como sendo necessárias e suficientes. Por exemplo, se for apresentada a seguinte regra "Se um avião sofre uma pane sobrevoando uma grande cidade, então o piloto deve pousar imediatamente". Nesse caso, há relações muito específicas contextualizadas na fórmula condicional que restringem sobremaneira as alternativas que poderiam ser consideradas. Assim, se for afirmado que o "piloto deve pousar imediatamente", dificilmente, as pessoas rejeitariam a conclusão de que um avião em pane sobrevoa a cidade. Nesse sentido, a ativação de exemplos alternativos seria inibida, concorrendo para a aceitação das inferências AC e NA. Por outro lado, sendo fraca a força associativa, alternativas mais fortemente associadas poderão ser disponibilizadas, uma vez que o próprio contexto seleciona na memória a alternativa que é mais provável. Isto contribuiria para uma interpretação condicional, qual seja a aceitação das inferências válidas MP e MT e a rejeição das inferências inválidas AC e NA, gerando uma maior quantidade de acertos.

\section{Método}

\section{Participantes}

Participaram, voluntariamente, 58 alunos de diferentes cursos de graduação do turno diurno de uma universidade local. Os participantes foram de ambos os sexos, sendo mulheres em sua maioria $(72,4 \%)$ com média de idade de 18,4 anos $(D P=$ $4,79)$. Os participantes não tinham treino em lógica formal.

\section{Delineamento}

Fatorial misto $2 \mathrm{x} 4$, onde a força associativa (forte e fraca) foi manipulada entre-sujeitos e tipo de inferência (MP, MT, AC e NA) foi manipulado intra-sujeitos. A variável dependente medida foi a porcentagem de acertos em tarefa semelhante ao experimento 1 . Os participantes foram designados aleatoriamente às condições experimentais.

\section{Materiais e Procedimentos}

Cada participante recebeu um conjunto de 36 problemas condicionais no formato "Se P, então Q" sendo que a proposição ' $\mathrm{P}$ ' se referiu à categoria contextualizada e a proposição ' $\mathrm{Q}$ ' incluiu um exemplo associado a esta categoria, sendo que para metade dos participantes o item era fortemente associado e para a outra metade, fracamente associado. O exemplo a seguir ilustra o tipo de problema que os participantes receberam na condição de membro fortemente associado à categoria:

Se é uma comemoração, então a bebida alcoólica servida deve ser a cerveja. Não é uma comemoração. O que deve ser concluído?

a bebida alcoólica servida deve ser a cerveja

a bebida alcoólica servida não deve ser a cerveja

não é possível concluir algo

As forças associativas entre exemplos e categorias foram retiradas das normas de associação semântica para categorias contextualizadas de Janczura (2005) que, por reduzirem o campo semântico, geram categorias com um conjunto menor de membros. Assim, nove categorias foram selecionadas para este estudo e tinham em média 6,6 itens $(D P=1,81)$. A média da força associativa dos membros fortemente e fracamente associados foram, respectivamente, $21,8 \%(D P=2,28)$ e $2,4 \%(D P=0,73)$.

As nove categorias contextualizadas foram apresentadas no formato impresso considerando-se os quatro casos lógicos MP, MT, AC e NA. Para cada nível da variável força associativa, forte e fraco, sendo apresentadas em 3 ordens randômicas. As instruções foram semelhantes ao estudo anterior. As coletas foram feitas com grupos de estudantes em salas de aula.

\section{Resultados e discussão}

A análise da variância indicou um efeito marginalmente significativo da força associativa $(F(1,56)=4,255, M S=$ 22,345, $p=0,0438)$, sendo observada uma média maior de acertos quando os membros eram fracamente associados (Média $=5,47)$ em contraste às médias de acertos para as inferências com membros fortemente associados (Média $=4,84)$. O teste $d$ de Cohen indicou efeito de magnitude pequena $(d=0,38)$. As 
diferenças nas médias indicam uma interpretação correta do de relações suficientes e não necessárias (Thompson, 1995). Efeito significativo também ocorreu em função do tipo de inferência $(F(3,168)=40,889, M S=216,598, p=0,000)$, sendo observadas as médias de acertos e desvios padrões ilustrados na tabela 2 .

O teste de Tukey $(D=0,57, p<0,05)$ indicou que a média de acertos para as inferências MP foi significativamente superior às demais médias. A título de realce, vale apontar que nesse estudo, a média de acertos para as inferências MP ficou muito próxima do número máximo de acertos (em média 7,95 em 8 acertos possíveis) em oposição à quase metade de acertos possíveis observados em relação à inferência $\mathrm{AC}$ (em média, 3,95 em 8). Esse resultado pode ser compreendido como uma tentativa em minimizar a sobrecarga da memória de trabalho pela construção de um número mínimo de modelos, conforme previam Johnson-Laird et al. (1992). Contudo, como pode ser observado na tabela 2 , houve relativa produção das inferências MT (em média, 4,94) as quais são raramente observadas em situações experimentais (Oaksford \& Chater, 1994). Contudo, o fato de ter havido uma diferença significativa entre as médias de acertos para as inferências MT e NA sugere interpretações bicondicionais, onde o padrão de desempenho é marcado pela ocorrência dos quatro tipos de inferências. Em resumo, a variação entre as médias ilustradas na tabela 2 sugerem que inferências baseadas em afirmativas (MP e AC) concorrem para a explicitação de um único modelo mental, enquanto inferências calcadas em negações (MT e NA) exigem a inserção de, pelo menos, mais um modelo. Essa racional é também consistente com os argumentos apresentados por Klauer e Oberauer (1995) quanto à relação entre número de modelos - principalmente se esses contem negações - e desempenho com condicionais: quanto mais modelos são necessários, pior o desempenho dada a sobrecarga da memória de trabalho. Por fim, os resultados apontam que a influência do fator força associativa é sensível ao contexto. Por exemplo, sem contexto delimitado, a categoria "bebida alcoólica" pode incluir como membro fortemente o "whiskey"; por outro lado, ao contextualizar a categoria como "bebida alcoólica servida em jantar íntimo", é provável que "vinho" seja mais provavelmente evocado. Isto sugere que a inclusão da força associativa em diferentes contextos é uma alternativa relevante para a compreensão da interação entre memória e raciocínio.

Tabela 2

Médias de acertos e desvios padrões para o tipo de inferência (Modus Ponens [MP], Modus Tollens [MT], Afirmação do Consequente [AC], Negação do Antecedente [NA])

\begin{tabular}{lcccc}
\hline \multicolumn{1}{c}{ Tipo de Inferência } & MP & MT & AC & NA \\
\hline Média & 7,95 & 4,94 & 3,95 & 3,78 \\
Desvio Padrão & 1,33 & 2,59 & 2,84 & 2,15 \\
\hline
\end{tabular}

\section{Discussão geral}

Os resultados dos dois experimentos evidenciaram uma interação entre características da estrutura representacional da informação na memória e o raciocínio condicional. Essa relação é consistente com a consideração de contraexemplos ativados de forma automática na memória semântica. No primeiro experimento, os efeitos de força associativa, especialmente, e do tamanho do grupo de associadas foram decisivos em relação às predições envolvendo as inferências MP e MT. De acordo com a teoria de modelos mentais (Johnson-Laird, 2001), esse padrão de desempenho incorre na aceitação de uma interpretação bicondicional baseada na necessidade e suficiência percebidas (Thompson, 1995). Contudo, o grande número de erros em relação às inferências $\mathrm{AC}$ não confirmou as expectativas quanto à facilitação do desempenho quando categorias e membros fracamente associados são utilizados. Uma hipótese para este resultado se refere ao fato de não ter sido controlada a força associativa dos membros utilizados como pistas e nomes de categorias geradas como resposta a estas pistas, isto é, a força associativa membro $\rightarrow$ categoria. Ou seja, a força associativa neste estudo indica apenas a probabilidade do membro frente à categoria, isto é, categoria $\rightarrow$ membro. Isto levanta a possibilidade de uma conexão ressonante (Nelson et al., 1998), sugerida pelo uso do par categoria-membro, onde é provável aceitar que não só pastor alemão é uma raça de cachorro, quando um bom exemplo de raça de cachorro é um pastor alemão, indicando uma relação bidirecional. Adicionalmente, uma variável apontada nos estudos de Nelson et al. (1998) que poderia contribuir para os resultados em relação às inferências AC é a conectividade. Nesse caso, membros alternativos podem não ter sido considerados devido à ausência de conexões interitens, isto é, entre o membro da categoria e outro item ou membro da mesma categoria, diminuindo, assim, a quantidade de ativação que um contraexemplo poderia receber. Desta maneira, a probabilidade de se considerar um exemplo alternativo seria reduzida. A ressonância e conectividade (Nelson et al., 1998) devem ser consideradas em estudos futuros para uma melhor compreensão dos efeitos do conhecimento sobre os processos de raciocínio, particularmente quando se configura a redução do campo semântico. Nesses casos, como evidenciou o segundo experimento, compreender os efeitos do contexto requer um delineamento mais refinado que inclua outras variáveis críticas na ativação da informação, tais como a ressonância, a conectividade e links indiretos de associação, além dos já investigados nesse estudo. Juntos, estes aspectos proporcionariam um quadro mais completo sobre como as relações em um determinado contexto seriam computadas, considerando-se a quantidade de ativação determinada por estes indexadores.

Adicionalmente, são relevantes estudos que permitam aprofundar a compreensão da relação entre processos ativos e automáticos de busca, ou mecanismos explícitos e implícitos de memória que possam afetar o raciocínio. Por exemplo, como apontam De Neys et al. (2005a), a contribuição relativa desses processos parece depender da natureza da informação. Segundo eles, estímulos contextualizados na relação categoria-membro, 
como os utilizados nesse estudo, envolvem mais processamento automático do que materiais "ad-hoc" que demandariam mais recursos da memória de trabalho para seu processamento. Nesse contexto é pertinente salientar que a ativação da informação na memória de longo-prazo não implica em carga ou sobrecarga de memória, no sentido estrito da palavra, uma vez que se assume o espalhamento (spreading) da ativação por uma rede semântica, criando uma representação implícita da informação, conforme defendem Nelson et al. (1998, 2007). Contudo, é possível que o executivo central da memória de trabalho possa, eventualmente, monitorar a ativação automática com o propósito de minimizar a ocorrência de erros. Por exemplo, De Neys et al. (2005b) encontraram evidências que a percepção de inconsistências lógicas podem inibir os processos de busca, inclusive, os automáticos. Outros aspectos contextualizados na relação memória-raciocínio que não foram explorados no presente estudo, também merecem certa atenção: as diferenças individuais, quer sejam de gênero ou sexo, idade ou recursos cognitivos. Usualmente, as pesquisas sobre o raciocínio humano tem atribuído pouca atenção às diferenças de gênero, sendo comuns amostras pouco balanceadas quanto ao percentual de homens e mulheres. É possível que esse fator exerça pouco efeito sobre o raciocínio condicional, exceto, talvez, em situações onde o conteúdo das premissas aluda às questões particulares dos universos masculino e feminino, evidenciando efeitos de especificidade do conteúdo. Vale lembrar que nesse estudo e em outros, como o de Quinn e Markovits (1998) - a maioria dos estímulos origina-se de procedimentos de coleta de normas, onde geralmente participam centenas de pessoas, homens e mulheres, cuja tarefa é evocar uma informação na presença de uma pista. O que resulta desse procedimento permite ao pesquisador prever o que pode ser ativado em tarefas futuras de memória, categorização ou raciocínio em função, por exemplo, da medida de força associativa. Nesse sentido, diferenças de gênero parecem não ser relevantes como possíveis fontes de variação. Contudo, uma perspectiva mais promissora diz respeito às diferenças entre indivíduos quanto aos recursos cognitivos. De Neys et al. (2005b) e García-Madruga et al. (2007) evidenciaram que os indivíduos que otimizam os recursos de suas memórias de trabalho apresentam melhor desempenho em tarefas de raciocínio. Finalmente, quanto às diferenças de idade, são mais comuns pesquisas que mapeiam diferenças nos processos de raciocínio em função do desenvolvimento cognitivo (e.g., Barrouillet \& Lecas, 1998), sendo mais escassas pesquisas comparativas entre adultos e idosos. Particularmente, esse é um campo de investigação assaz promissor, dadas as diferenças no sistema de memória decorrentes do envelhecimento normal. Adicionalmente, pesquisas comparativas como essas permitiriam estabelecer de forma mais inequívoca o papel relativo dos processos de busca da informação relevantes para o raciocínio condicional.

\section{Referências}

Barrouillet, P., Gauffroy, C., \& Lecas, J-F. (2008). Mental models and the suppositional account of conditionals. Psychological Review, 115, 760-771. Barrouillet, P., \& Lecas, J-F. (1998). How can mental models theory account for content effects in conditional reasoning? A developmental perspective. Cognition, 67, 209-253.

Bonatti, L. (1994). Propositional reasoning by model? Psychological Review, $101,725-733$.

Braine, M.D.S. (1978). On the relation between the natural logic of reasoning and standard logic. Psychological Review, 85, 1-21.

Bucci, W. (1978). The interpretation of universal affirmative propositions. Cognition, 6, 55-77.

Byrne, R.M.J. (1989). Suppressing valid inferences with conditionals. Cognition, $31,61-83$

Cummins, D.D. (1995). Naive theories and causal deduction. Memory \& Cognition, 23, 646-658

Cummins, D.D., Lubart, T., Alksnis, O., \& Rist, R. (1991). Conditional reasoning and causation. Memory \& Cognition, 19, 274-282.

De Neys, W., Schaeken, W., \& d'Ydewalle, G. (2002). Causal conditional reasoning and semantic memory retrieval: A test of the semantic memory framework. Memory \& Cognition, 30, 908-920.

De Neys, W., Schaeken, W., \& d'Ydewalle, G. (2005a). Working memory and counterexamples retrieval for causal conditionals. Thinking \& Reasoning, $11,123-150$

De Neys, W., Schaeken, W., \& d'Ydewalle, G. (2005b). Working memory and everyday conditional reasoning: Retrieval and inhibition of stored counterexamples. Thinking \& Reasoning, 11, 349-381.

Evans, J.St.B.T. (1993). The mental model theory of conditional reasoning: Critical appraisal and revision. Cognition, 48, 1-20.

Evans, J.St.B.T., Clibbens, J., \& Rood, B. (1995). Bias in conditional inference: Implications for mental models and mental logic. The Quarterly Journal of Experimental Psychology, 48A, 644-670.

Evans, J.St.B.T., \& Twyman-Musgrove, J. (1998). Conditional reasoning with inducements and advice. Cognition, 69, B11-B16.

García-Madruga, J.A., Gutiérrez, F., Carriedo, N., Luzón, J.M., \& Vila, J.O. (2007). Mental models in propositional reasoning and workingmemory's central executive. Thinking \& Reasoning, 13, 370-393.

Goodwin, G.P., \& Johnson-Laird, P.N. (2005). Reasoning about relations. Psychological Review, 112, 468-493.

Janczura, G.A. (1996). Normas associativas para 69 categorias semânticas. Psicologia: Teoria e Pesquisa, 12, 237-244

Janczura, G.A. (2005). Contexto e normas de associação para palavras: a redução do campo semântico. Paidéia: Caderno de Psicologia, 15, 417-425.

Johnson-Laird, P.N. (2001). Mental models and deduction. Trends in Cognitive Science, 10, 434-442.

Johnson-Laird, P.N., \& Byrne, R.M.J. (2002). Conditionals: A theory of meaning, pragmatics and inference. Psychological Review, 109, 646-678.

Johnson-Laird, P.N., Byrne, R.M.J., \& Schaeken, W. (1992). Propositional reasoning by model. Psychological Review, 99, 411-439.

Klauer, K.C., \& Oberauer, K. (1995). Testing the mental models theory of propositional reasoning. The Quarterly Journal of Experimental Psychology, 48A, 671-687.

Legrenzi, P., Girotto, V., \& Johnson-Laird, P.N. (1993). Focussing in reasoning and decision making. Cognition, 49, 37-66.

Nelson, D. L., Dyrdal, G. M., \& Goodmon, L.B. (2005). What is pre-existing strength? Predicting free association probabilities, similarity ratings, and cued recall probabilities. Psychonomic Bulletin \& Review, 12, 711-719.

Nelson, D. L., Fisher, S., \& Akirmak, U. (2007). How does implicitly activated and explicitly acquired knowledge contribute to the effectiveness of extralist cues? Memory \&Cognition, 35, 1892-1904.

Nelson, D. L., McEvoy, C. L., \& Pointer, L. (2003). Spreading activation or spooky action at a distance? Journal of Experimental Psychology: Learning, Memory and Cognition, 29, 42-52.

Nelson, D.L., McKinney, V.M., Gee, N.R., \& Janczura, G.A. (1998). Interpreting the influence of implicitly activated memories on recall and recognition. Psychological Review, 105, 299-324. 
Nelson, D.L., Schreiber, T.A., \& McEvoy, C.L. (1992). Processing implicit and explicit representations. Psychological Review, 99, 322-348.

Oaksford, M., \& Chater, N. (1994). A rational analysis of the selection task as optimal data selection. Psychological Review, 101, 608-631.

Pollard, P. (1982). Human Reasoning: Some possible effects of availability. Cognition, 12, 65-96.

Quinn, S., \& Markovits, H. (1998). Conditional reasoning, causality, and the structure of semantic memory: Strength of association as a predictive factor for content effects. Cognition, 68, B93-B101.
Rips, L.J. (1983). Cognitive processes in propositional reasoning. Psychological Review, 90, 38-71.

Schroyens, W., \& Schaeken, W. (2008). Deductive rationality in validating and testing conditional inferences. Canadian Journal of Experimental Psychology, 62, 163-173.

Thompson, V.A. (1995). Conditional reasoning: The necessary and sufficient conditions. Canadian Journal of Experimental Psychology, 49, 1-58.

Wason, P.C. (1968). Reasoning about a rule. The Quarterly Journal of Experimental Psychology, 20, 273-281.

Goiara Mendonça de Castilho, doutor em Psicologia/Psicologia Cognitiva pela Universidade de Brasília, é professor Adjunto 1 na Universidade de Brasília. Endereço: Departamento de Processos Psicológicos Básicos (PPB), Instituto de Psicologia (IP) Instituto Central de Ciências - Campus Darcy Ribeiro. Endereço para correspondência: Universidade de Brasília (UnB). Asa Norte. 70910-900 - Brasília, DF-Brasil. Tel: (61) 3107-6959 (UnB), (61) 9153-7729 (Cel). E-mail: goiacas@unb.br ou goiaracastilho@gmail.com

Gerson Américo Janczura, doutor e Ph.D em Psicologia Cognitiva Experimental pela University of South Florida, é professor Associado na Universidade de Brasília. E-mail: janczura@unb.br 